\title{
Synthesis and Characterization of Symmetrical and Unsymmetrical Analogues of 1,2-bis[bis(4-chlorophenyl) Methyl] Diselane
}

\author{
Rajeev Kumar \\ Department of Environment Studies, Panjab University, India
}

Copyright (C) 2015 Horizon Research Publishing All rights reserved.

\begin{abstract}
The 'living' species selenide anion $\mathrm{Se}_{2}{ }^{2-}$, generated in situ by the reduction of 1,2-bis(bis(4-chlorophenyl)methyl)diselane with sodium borohydride, was treated with various organic halides to afford synthetically important organoselenium compounds including $\alpha, \omega$-diselenoethers and selenides bearing ester functionality, under mild and neutral conditions. These compounds have been characterized analytically with the help of various spectroscopic techniques viz., IR, multinuclear NMR $\left({ }^{1} \mathrm{H},{ }^{13} \mathrm{C},{ }^{77} \mathrm{Se}\right)$ and mass spectrometry.
\end{abstract}

Keywords Selenide, Diselenide, Symmetrical Diselenide, Unsymmetrical Selenide, Selenoether

\section{Introduction}

In recent years, the synthesis of organoselenium compounds have become an increasingly popular area in the field of synthetic reagents and intermediates in organic synthesis [1,2]. Specifically, synthetic routes for the introduction of seleno-substituents into organic molecule which are the building blocks for the synthesis of modified selenocarbohydrates, selenoaminoacids and selenoproteins have attracted much attention due to their interesting structural and biological properties [3]. Literature is inundated with several synthetic methods for the synthesis of selenide anion, the more important of which include the use of reduction of diorganyl diselenide with sodium [4], samarium diiodide [5], Sm/TMSCl [6] and catalyzed system $\mathrm{Cu}_{2} \mathrm{O} / \mathrm{Mg} /$ bpy [7]. Moreover, Grignard reagents [8,9] also react with selenium to give equivalent species of selenide anions.

A scrutiny of literature, pertinent to the chemistry of diaryl methyl selenium/tellurium compounds and their applications deserve a special mention here. Recently, it has been reported that diphenyl methyl selenocynate $\left(\mathrm{Ph}_{2} \mathrm{CHSeCN}\right)$ plays a protective role against carbon tetrachloride induced heptatoxicity in mice [10]. Margolis and Pittman[11] prepared bis(diphenylmethyl) diselenide by using two methodologies one involve the reduction of benzophenone using hydrogen selenide and another by treating diphenyl methyl bromide with sodium hydrogen selenide. Okama et al. [12] reported the synthesis of diphenyl methyl diselenide in satisfactory yields by the reduction of seleonocarbonyl intermediate with $\mathrm{NaBH}_{4}$. Kamigata et al. [13] have shown the formation of methyl diphenylmethyl selenide and benzyl diphenylmethyl selenide in low yields by the reaction of 1,3-bis(alkylseleno)allenes and diphenyl diazomethane in refluxing benzene.

In the light of our experimental interests $[14,15]$ on the aromatic selenium and tellurium compounds, we report in this communication the synthesis and characterization of 1,2-bis(bis(4-chlorophenyl)methyl)diselane and further a convenient method for the reductive cleavage of Se-Se bond of 1,2-bis(bis(4-chlorophenyl) methyl) diselane with sodium borohydride to generate selenide anion. Sodium borohydride, as a reducing agent capable of reducing many functionalities like nitrile, ester, carbonyl, epoxide and double bonds. Ethanolic sodium borohydride has been widely used to generate sodium selenide ions in a protic media. The complete reduction of diorganyl diselenides leads to the formation of organyl selenolates $[16,17]$, which are colorless in solution. It has been found that selenide anion, a 'living' species, reacted smoothly with active organic halides to give the designed symmetrical/unsymmetrical selenium derivatives in good yields.

\section{Materials and Methods}

\subsection{General Methods}

All the reactions were carried out under dry and deoxygenated nitrogen atmosphere. Selenium/Tellurium was estimated by standard methods. Sodium borohydride (Loba), selenium (Hi-media) and tellurium (Aldrich) were stored in dessicator prior to use. 4,4'-Dichloro diphenylmethyl chloride was prepared by literature method. All other 
chemicals were of analytical grade and used without further purification. Ethanol was freshly distilled and dehydrated under sodium metal. Separation and purification of the compounds was done by column chromatography performed on activated silica gel (100-120) using hexane as eluant.

${ }^{1} \mathrm{H},{ }^{13} \mathrm{C}\left\{{ }^{1} \mathrm{H}\right\}$ NMR was recorded in $\mathrm{CDCl}_{3}$ using $\mathrm{Me}_{4} \mathrm{Si}$ as an internal standard using Jeol AL $300 \mathrm{MHz}$ spectrometer. ${ }^{77} \mathrm{Se}\left\{{ }^{1} \mathrm{H}\right\}$ NMR was recorded on the same instrument using $\mathrm{Me}_{2} \mathrm{Se}$ as an external reference. Infrared spectra were obtained either between $\mathrm{KBr}$ pellets as neat liquids or as compressed $\mathrm{KBr}$ discs in case of solid compounds on Perkin-Elmer model-1430 spectrophotometer. Mass spectra were obtained on a micro Q-TOF mass spectrometer.

\subsection{Synthesis of 1,2-bis[bis(4-chlorophenyl) methyl] Diselane}

To a vigorously stirred mixture of powdered sodium hydroxide ( $3.0 \mathrm{~g}, 75 \mathrm{mmol})$, elemental selenium $(4.0 \mathrm{~g}, 50$ $\mathrm{mmol})$ and dimethylformamide $(30 \mathrm{ml}), 100 \%$ hydrazine hydrate was added slowly. After stirring for nearly $6 \mathrm{~h}$ at room temperature, a solution of 4,4'-dichloro diphenylmethyl chloride $(100 \mathrm{mmol})$ dissolved in $15 \mathrm{ml}$ DMF was added dropwise. When the color of the solution became light yellow, it was diluted with about $250 \mathrm{ml}$ of distilled water and extracted in dichloromethane $(3 \times 50 \mathrm{ml})$. The organic layer was washed with $6 \mathrm{~N} \mathrm{HCl}$ followed by distilled water and then dried over anhydrous sodium sulfate. Solvent was evaporated on a rotary-evaporator and the product was subjected to purification on a silica column using hexane as the eluant.

2.2.1. 1,2-bis(bis(4-chlorophenyl)methyl)diselane $\left[\left(\mathrm{ClC}_{6} \mathrm{H}_{4}\right)_{2} \mathrm{CHSe}\right]_{2}(\mathbf{1})$. Yellow crystalline solid ( $89 \%$ yield). m.p. $92-95^{\circ} \mathrm{C}$. IR $\left(v_{\max } / \mathrm{cm}^{-1}, \mathrm{KBr}\right): 3025,2925,2584,2357$, 1899, 1779, 1660, 1591, 1488, 1402, 1215, 1176, 1092, 1014, 925, 787, 681, 521, 499, 414. MS-EI, m/e (RI, assignment): $409\left(15,\left[\mathrm{C}_{14} \mathrm{H}_{10} \mathrm{Se}_{2} \mathrm{Cl}_{2}\right]^{+}\right), 397\left(6,\left[\mathrm{C}_{13} \mathrm{H}_{8} \mathrm{Se}_{2} \mathrm{Cl}_{2}\right]^{+}+2\right), 234$ $\left(11,\left[\mathrm{C}_{13} \mathrm{H}_{9} \mathrm{Cl}_{2}\right]^{+}-1\right), 204\left(8,\left[\mathrm{C}_{7} \mathrm{H}_{5} \mathrm{SeCl}\right]^{+}-2\right) . \mathrm{NMR}\left({ }^{1} \mathrm{H}, 300\right.$ $\left.\mathrm{MHz}, \mathrm{CDCl}_{3}\right): \delta 7.30-7.23(\mathrm{~m}, 8 \mathrm{H}), 5.71(\mathrm{~s}, 1 \mathrm{H})$. NMR $\left({ }^{13} \mathrm{C}\left\{{ }^{1} \mathrm{H}\right\}, 75 \mathrm{MHz}, \mathrm{CDCl}_{3}\right): \delta 139.71,139.15,138.94$, $138.90,138.48,135.58,133.91,133.69,133.60,131.45$, $131.17,130.29,129.99,128.74,128.24,127.90,49.71$; $\operatorname{NMR}\left({ }^{77} \mathrm{Se}\left\{{ }^{1} \mathrm{H}\right\}, 57 \mathrm{MHz}, \mathrm{CDCl}_{3}\right): \delta 528.5$.

\subsection{Synthesis of Symmetrical 1,2-bis[bis(4-chloro phenyl) methyl] Alkanes}

To a $3.15 \mathrm{gm} \quad(5 \mathrm{mmol})$ solution of 1,2-bis(bis(4-chlorophenyl)methyl)diselane in $50 \mathrm{ml}$ $\mathrm{C}_{2} \mathrm{H}_{5} \mathrm{OH}-\mathrm{DMF}(3: 2)$ was added $0.456 \mathrm{gm}(12 \mathrm{mmol})$ of $\mathrm{NaBH}_{4}$ in three parts with continuous stirring at room temperature in 30 mintues. After $3 \mathrm{~h}$ of stirring, $10 \mathrm{mmol}$ of alkylating agent, $\mathrm{X}\left(\mathrm{CH}_{2}\right)_{\mathrm{n}} \mathrm{X}$, diluted with equal volume of DMF was added dropwise at room temperature. Completion of reaction was monitored with the help of TLC. Extraction was done with dichloromethane after evaporating ethanol under vacuum. The organic layer was washed repeatedly with distilled water $(3 \times 40 \mathrm{ml})$, dried over anhydrous
$\mathrm{Na}_{2} \mathrm{SO}_{4}$. Solvent is evaporated on a rotary-evaporator and the product is subjected to purification on a silica column using hexane as the eluant.

2.3.1. Bis[bis(4-chlorophenyl) methylselanyl] methane, [ $\left.\left(\mathrm{ClC}_{6} \mathrm{H}_{4}\right)_{2} \mathrm{CHSe}\right]_{2} \mathrm{CH}_{2}$ (2a). Yellow solid (74\% yield). m.p. 58-60 ${ }^{\circ} \mathrm{C}$. IR $\left(v_{\max } / \mathrm{cm}^{-1}, \mathrm{KBr}\right): 3435,2954,2853,2360$, $1639,1488,1092,908,787,732,525$. NMR $\left({ }^{1} \mathrm{H}, 300 \mathrm{MHz}\right.$, $\left.\mathrm{CDCl}_{3}\right): \delta 7.25-7.14(\mathrm{~m}, 8 \mathrm{H}), 5.38(\mathrm{~s}, 1 \mathrm{H}), 3.09(\mathrm{~s}, 2 \mathrm{H})$. NMR $\left({ }^{13} \mathrm{C}\left\{{ }^{1} \mathrm{H}\right\}, 75 \mathrm{MHz}, \mathrm{CDCl}_{3}\right): \delta 138.77,133.32,131.02$, 130.21, 129.91, 129.10, 128.48, 127.97, 48.50, 48.14.

2.3.2. [2-\{bis(4-chlorophenyl) methylselanyl ethaneselanyl] bis(4-chlorophenyl)methane

$\left[\left(\mathrm{ClC}_{6} \mathrm{H}_{4}\right)_{2} \mathrm{CHSe}_{2}\left(\mathrm{CH}_{2}\right)_{2}\right.$ (2b). Yellow solid (89\% yield). m.p $80-82^{\circ} \mathrm{C}$. IR $\left(v_{\max } / \mathrm{cm}^{-1}, \mathrm{KBr}\right): 3420,2925,2853,2347$, 1632，1458，1397，906，785，758 . MS-EI， m/e (RI, assignment): $\quad 646\left(5, \quad\left[\mathrm{C}_{27} \mathrm{H}_{20} \mathrm{Se}_{2} \mathrm{Cl}_{4}\right]^{+}+1\right), \quad 518 \quad(20$, $\left.\left[\mathrm{C}_{28} \mathrm{H}_{20} \mathrm{Se}_{2}\right]^{+}+2\right), 513\left(28, \quad\left[\mathrm{C}_{22} \mathrm{H}_{18} \mathrm{Se}_{2} \mathrm{Cl}_{2}\right]^{+}-2\right), 330$ (66, $\left.\left[\mathrm{C}_{14} \mathrm{H}_{11} \mathrm{SeCl}_{2}\right]^{+}\right), \quad 325 \quad\left(7, \quad\left[\mathrm{C}_{10} \mathrm{H}_{10} \mathrm{Se}_{2} \mathrm{Cl}\right]^{+}\right), \quad 316 \quad(6$, $\left.\left[\mathrm{C}_{13} \mathrm{H}_{9} \mathrm{SeCl}_{2}\right]^{+}\right), 236\left(6,\left[\mathrm{C}_{13} \mathrm{H}_{9} \mathrm{SeCl}_{2}\right]^{+}\right), 212\left(9,\left[\mathrm{C}_{4} \mathrm{H}_{6} \mathrm{Se}_{2}\right]^{+}\right)$. $\operatorname{NMR}\left({ }^{1} \mathrm{H}, 300 \mathrm{MHz}, \mathrm{CDCl}_{3}\right): \delta 7.21-7.16(\mathrm{~m}, 8 \mathrm{H}), 5.15(\mathrm{~s}$, 1H), 2.51 (s, 4H). NMR $\left({ }^{13} \mathrm{C}\left\{{ }^{1} \mathrm{H}\right\}, 75 \mathrm{MHz}, \mathrm{CDCl}_{3}\right): \delta$ 139.06, 135.61, 131.43, 131.09, 129.60, 128.85, 52.35, 51.92, 50.56 .

2.3.3. [3-\{bis(4-chlorophenyl) methylselanyl\} propylselanyl] bis(4-chlorophenyl) methane, $\left[\left(\mathrm{ClC}_{6} \mathrm{H}_{4}\right)_{2} \mathrm{CHS}\right.$ e] ${ }_{2}\left(\mathrm{CH}_{2}\right)_{3}(\mathbf{2 c})$. Yellow semisolid $(63 \%$ yield $)$. IR $\left(v_{\max } / \mathrm{cm}^{-}\right.$ 1 , KBr): 3410, 3137, 2924, 2856, 2360, 1591, 1400, 1089, 1016, 909, 736. NMR $\left({ }^{1} \mathrm{H}, 300 \mathrm{MHz}, \mathrm{CDCl}_{3}\right): \delta$ 7.27-7.21 (m, $8 \mathrm{H}), 5.25(\mathrm{~s}, 1 \mathrm{H}), 3.43-3.23(\mathrm{t}, J=6.3 \mathrm{~Hz}, 2 \mathrm{H}), 2.49-2.45(\mathrm{t}$, $J=6.9 \mathrm{~Hz}, 2 \mathrm{H}), 2.04-1.96(\mathrm{~m}, 2 \mathrm{H}) . \mathrm{NMR}\left({ }^{13} \mathrm{C}\left\{{ }^{1} \mathrm{H}\right\}, 75 \mathrm{MHz}\right.$, $\left.\mathrm{CDCl}_{3}\right): \delta 139.51,133.24,133.17,130.81,129.96,129.89$, $128.82,128.38,127.98,46.99,45.42,32.66,32.55,24.06$. $\operatorname{NMR}\left({ }^{77} \mathrm{Se}\left\{{ }^{1} \mathrm{H}\right\}, 57 \mathrm{MHz}, \mathrm{CDCl}_{3}\right): \delta 339.12$.

\subsection{Synthesis of Unsymmetrical 1,2-bis(bis(4-chloro phenyl) methyl)selenides}

To a $3.15 \mathrm{gm} \quad(5 \mathrm{mmol})$ solution of 1,2-bis(bis(4-chlorophenyl)methyl)diselane in $50 \mathrm{ml}$ $\mathrm{C}_{2} \mathrm{H}_{5} \mathrm{OH}-\mathrm{DMF}$ (3:2) was added $0.456 \mathrm{gm}(12 \mathrm{mmol})$ of $\mathrm{NaBH}_{4}$ in three parts with continuous stirring at room temperature in 30 minutes. After $3 \mathrm{~h}$ of stirring, $10 \mathrm{mmol}$ of alkylating agent (RX) diluted with equal volume of DMF was added dropwise at room temperature. Completion of reaction was monitored with TLC. Extraction is done with dichloromethane after evaporating ethanol under vacuum. The organic layer is washed repeatedly with distilled water $(3 \times 40 \mathrm{ml})$, dried over anhydrous $\mathrm{Na}_{2} \mathrm{SO}_{4}$. Solvent is evaporated on a rotary-evaporator and the product is subjected to purification on a silica column using hexane as the eluant.

2.4.1. Bis(4-chlorophenyl)methyl(methyl)selane, $\left[\left(\mathrm{ClC}_{6} \mathrm{H}_{4}\right)_{2} \mathrm{CHSeCH}_{3}\right](\mathbf{3 d})$. Yellow oil (54\% yield). IR ( $v_{\max }$ $\left./ \mathrm{cm}^{-1}, \mathrm{KBr}\right): 3395,2924,2856,2369,2347,1489,1092,909$, 793. NMR $\left({ }^{1} \mathrm{H}, 300 \mathrm{MHz}, \mathrm{CDCl}_{3}\right): \delta 7.22-7.12(\mathrm{~m}, 8 \mathrm{H}), 5.12$ (s, 1H), 1.76(s, 3H). NMR $\left({ }^{13} \mathrm{C}\left\{{ }^{1} \mathrm{H}\right\}, 75 \mathrm{MHz}, \mathrm{CDCl}_{3}\right): \delta$ $139.68,133.88,130.89,129.71,128.92,127.90,46.25,6.39$. $\left({ }^{77} \mathrm{Se}\left\{{ }^{1} \mathrm{H}\right\}, 57 \mathrm{MHz}, \mathrm{CDCl}_{3}\right): \delta 266.8$ 
2.4.2. Bis(4-chlorophenyl)methyl(ethyl) selane, [( $\left.\left.\mathrm{ClC}_{6} \mathrm{H}_{4}\right)_{2} \mathrm{CHSeCH}_{2} \mathrm{CH}_{3}\right]$ (3e). Light yellow solid $(78 \%$ yield). m.p 50-53 ${ }^{\circ}$ C.IR $\left(v_{\max } / \mathrm{cm}^{-1}, \mathrm{KBr}\right): 3399,2924,1665$, 1594, 1490, 1401, 1174, 1091, 908, 850, 736, 521. MS-EI, m/e (RI, assignment): $347\left(4,\left[\mathrm{C}_{15} \mathrm{H}_{14} \mathrm{SeCl}_{2}\right]^{+}+2\right), 331$ (6, $\left.\left[\mathrm{C}_{14} \mathrm{H}_{12} \mathrm{SeCl}_{2}\right]^{+}\right), 330 \quad\left(24, \quad\left[\mathrm{C}_{14} \mathrm{H}_{11} \mathrm{SeCl}_{2}\right]^{+}\right), 318 \quad(7$, $\left.\left[\mathrm{C}_{13} \mathrm{H}_{9} \mathrm{SeCl}_{2}\right]^{+}+2\right), \quad 274 \quad\left(47, \quad\left[\mathrm{C}_{15} \mathrm{H}_{4} \mathrm{Se}\right]^{+}\right), \quad 246 \quad(9$, $\left.\left[\mathrm{C}_{13} \mathrm{H}_{11} \mathrm{Se}\right]^{+}+1\right), 229\left(6,\left[\mathrm{C}_{14} \mathrm{H}_{12} \mathrm{SeCl}_{2}\right]^{+}\right), \operatorname{NMR}\left({ }^{1} \mathrm{H}, 300\right.$ $\left.\mathrm{MHz}, \mathrm{CDCl}_{3}\right): \delta 7.23-6.98(\mathrm{~m}, 8 \mathrm{H}), 5.51(\mathrm{~s}, 1 \mathrm{H}), 2.36-2.25(\mathrm{q}$, $J=7.5 \mathrm{~Hz}, 2 \mathrm{H}), 1.17-1.24(\mathrm{t}, J=7.5 \mathrm{~Hz}, 3 \mathrm{H})$. NMR $\left({ }^{13} \mathrm{C}\left\{{ }^{1} \mathrm{H}\right\}\right.$, $\left.75 \mathrm{MHz}, \mathrm{CDCl}_{3}\right): \delta 141.90,139.74,133.52,129.96,128.64$, 127.87, 126.96, 46.35, 19.58, 15.22. $\left({ }^{77} \mathrm{Se}\left\{{ }^{1} \mathrm{H}\right\}, 57 \mathrm{MHz}\right.$, $\left.\mathrm{CDCl}_{3}\right): \delta 396.5$

2.4.3.Bis(4-chlorophenyl)methyl(allyl)selane, $\left[\left(\mathrm{ClC}_{6} \mathrm{H}_{4}\right)_{2}\right.$ $\left.\mathrm{CHSeCH}_{2} \mathrm{CHCH}_{2}\right]$ (3f). Colorless viscous oil (52\% yield). IR $\left(v_{\max } / \mathrm{cm}^{-1}, \mathrm{KBr}\right): 3432,2924,2347,1897,1489,1399$, 1259, 1092, 1016, 916, 786, 523. NMR $\left({ }^{1} \mathrm{H}, 300 \mathrm{MHz}\right.$, $\left.\mathrm{CDCl}_{3}\right): \delta 7.26-7.10(\mathrm{~m}, 8 \mathrm{H}), 5.85-5.71(\mathrm{~m}, 1 \mathrm{H}), 5.12(\mathrm{~s}, 1 \mathrm{H})$, 4.98-4.89 (d, $J=9.9 \mathrm{~Hz}, 1 \mathrm{H}), 4.77-4.71(\mathrm{~d}, J=16.8 \mathrm{~Hz}, 1 \mathrm{H})$, 2.93-2.85 (d, $J=7.5 \mathrm{~Hz}, 2 \mathrm{H})$. NMR $\left({ }^{13} \mathrm{C}\left\{{ }^{1} \mathrm{H}\right\}, 75 \mathrm{MHz}\right.$, $\left.\mathrm{CDCl}_{3}\right): \delta 139.73,133.76,133.16,130.20,128.92,128.85$, 128.79, 126.96, 46.45, 44.84. NMR $\left({ }^{77} \mathrm{Se}\left\{{ }^{1} \mathrm{H}\right\}, 57 \mathrm{MHz}\right.$, $\left.\mathrm{CDCl}_{3}\right): \delta 459.62$.

2.4.4. Bis(4-chlorophenyl)methyl)(butyl) selane, [ $\left.\left(\mathrm{ClC}_{6} \mathrm{H}_{4}\right)_{2} \mathrm{CH} \mathrm{SeCH} \mathrm{CH}_{2} \mathrm{CH}_{2} \mathrm{CH}_{3}\right]$ (3g). Yellow oil liquid (63\% yield). IR ( $\left.v_{\max } / \mathrm{cm}^{-1}, \mathrm{KBr}\right): 3410,2928,2361,1628$, 1484, 1092, 793. NMR $\left({ }^{1} \mathrm{H}, 300 \mathrm{MHz}, \mathrm{CDCl}_{3}\right): \delta$ 7.28-7.17 $(\mathrm{m}, 8 \mathrm{H}), 5.19(\mathrm{~s}, 1 \mathrm{H}), 2.36-2.27(\mathrm{t}, J=7.2 \mathrm{~Hz}, 2 \mathrm{H}), 1.57-1.42$ $(\mathrm{m}, 2 \mathrm{H}), 1.28-1.17(\mathrm{~m}, 2 \mathrm{H}), 0.75-0.87(\mathrm{t}, J=7.2 \mathrm{~Hz}, 3 \mathrm{H})$. $\mathrm{NMR}\left({ }^{13} \mathrm{C}\left\{{ }^{1} \mathrm{H}\right\}, 75 \mathrm{MHz}, \mathrm{CDCl}_{3}\right): \delta 151.25,141.01,140.71$, 139.91, 129.96, 128.83, 119.39, 45.01, 31.98, 25.88, 13.66.

2.4.5. Ethyl2-[bis(4-chlorophenyl)methylselanyl]acetate, $\left[\left(\mathrm{ClC}_{6} \mathrm{H}_{4}\right)_{2} \mathrm{CHSeCH}_{2} \mathrm{COOCH}_{2} \mathrm{CH}_{3}\right]$ (3h).Yellow oil $(78 \%$ yield). IR ( $\left.v_{\max } / \mathrm{cm}^{-1}, \mathrm{KBr}\right): 3422,2926,1731,1489,1404$, 1363, 1265, 1256, 1093, 1033, 793. MS-EI, m/e (RI, assignment): $359 \quad\left(34, \quad\left[\mathrm{C}_{16} \mathrm{H}_{16} \mathrm{SeCl}_{2}\right]^{+}\right), \quad 357 \quad(6$, $\left.\left[\mathrm{C}_{14} \mathrm{H}_{12} \mathrm{SeCl}_{2}\right]^{+}\right), \quad 330 \quad\left(16, \quad\left[\mathrm{C}_{14} \mathrm{H}_{11} \mathrm{SeCl}_{2}\right]^{+}\right), 288 \quad(6$, $\left.\left[\mathrm{C}_{16} \mathrm{H}_{16} \mathrm{Se}\right]^{+}\right), \quad 279 \quad\left(22, \quad\left[\mathrm{C}_{16} \mathrm{H}_{16} \mathrm{Cl}_{2}\right]^{+}\right), 273 \quad(12$, $\left.\left[\mathrm{C}_{15} \mathrm{H}_{13} \mathrm{Se}\right]^{+}+1\right), 260\left(7,\left[\mathrm{C}_{14} \mathrm{H}_{10} \mathrm{Se}\right]^{+}\right)$. NMR $\left({ }^{1} \mathrm{H}, 300 \mathrm{MHz}\right.$, $\left.\mathrm{CDCl}_{3}\right): \delta 7.37-7.12(\mathrm{~m}, 8 \mathrm{H}), 5.53(\mathrm{~s}, 1 \mathrm{H}), 4.08-4.01(\mathrm{q}, J=$ $7.2 \mathrm{~Hz}, 2 \mathrm{H}), 2.82(\mathrm{~s}, 2 \mathrm{H}), 1.23-1.16(\mathrm{t}, J=7.2 \mathrm{~Hz}, 3 \mathrm{H})$. NMR $\left({ }^{13} \mathrm{C}\left\{{ }^{1} \mathrm{H}\right\}, 75 \mathrm{MHz}, \mathrm{CDCl}_{3}\right): \delta 170.74,138.72,138.68$, $133.48,130.14,129.02,128.84,128.04,61.11,24.44,14.32$. NMR $\left({ }^{77} \mathrm{Se}\left\{{ }^{1} \mathrm{H}\right\}, 57 \mathrm{MHz}, \mathrm{CDCl}_{3}\right): \delta 251.13$.

2.4.6.

Ethyl3-[bis(4-chlorophenyl)methylselanyl]propanoate, [ $\left.\left(\mathrm{ClC}_{6} \mathrm{H}_{4}\right)_{2} \mathrm{CHSeCH}_{2} \mathrm{CH}_{2} \mathrm{COOCH}_{2} \mathrm{CH}_{3}\right]$ (3i). Yellow oil (65\% yield). IR ( $\left.v_{\max } / \mathrm{cm}^{-1}, \mathrm{KBr}\right): 3426,2928,2362,1735,1214$, 1089, 793. NMR $\left({ }^{1} \mathrm{H}, 300 \mathrm{MHz}, \mathrm{CDCl}_{3}\right): \delta 7.27-7.17(\mathrm{~m}, 8 \mathrm{H})$, $5.31(\mathrm{~s}, 1 \mathrm{H}), 4.09-4.02(\mathrm{q}, J=7.2 \mathrm{~Hz}, 3 \mathrm{H}), 2.56-2.51(\mathrm{~m}, 4 \mathrm{H})$, 1.21-1.17 (t, $J=7.2 \mathrm{~Hz}, 3 \mathrm{H})$. NMR $\left({ }^{13} \mathrm{C}\left\{{ }^{1} \mathrm{H}\right\}, 75 \mathrm{MHz}\right.$, $\left.\mathrm{CDCl}_{3}\right): \delta 171.70,141.01,140.11,133.01,130.11,128.79$, $128.74,128.72,127.37,60.55,47.19,35.14,19.72,14.42$. NMR $\left({ }^{77} \mathrm{Se}\left\{{ }^{1} \mathrm{H}\right\}, 57 \mathrm{MHz}, \mathrm{CDCl}_{3}\right): \delta 305.58$.

2.4.7.

Ethyl4-[bis(4-chlorophenyl)methylselenyl]butanoate,
[( $\left.\left(\mathrm{ClC}_{6} \mathrm{H}_{4}\right)_{2} \mathrm{CHSeCH}_{2} \mathrm{CH}_{2} \mathrm{CH}_{2} \mathrm{COOCH}_{2} \mathrm{CH}_{3}\right]$ (3j). Yellow oil $(50 \%$ yield $)$. IR $\left(v_{\max } / \mathrm{cm}^{-1}, \mathrm{KBr}\right): 3432,3028,2925,2250$, 2076, 1720, 1599, 1490, 1451, 1408, 1203, 1090, 104, 909, 789, 735, 553, 502. NMR $\left({ }^{1} \mathrm{H}, 300 \mathrm{MHz}, \mathrm{CDCl}_{3}\right): \delta$ $7.25-7.118(\mathrm{~m}, 8 \mathrm{H}), 5.22(\mathrm{~s}, 1 \mathrm{H}), 4.08-3.96(\mathrm{q}, J=6.9 \mathrm{~Hz}$, $2 \mathrm{H}), 3.51-3.47(\mathrm{t}, J=6.9 \mathrm{~Hz}, 2 \mathrm{H}), 2.92-2.22(\mathrm{t}, J=6.9 \mathrm{~Hz}$, $2 \mathrm{H}), 1.85-1.75(\mathrm{~m}, 2 \mathrm{H}), 1.18-1.13(\mathrm{t}, J=6.9 \mathrm{~Hz}, 3 \mathrm{H})$. NMR $\left({ }^{13} \mathrm{C}\left\{{ }^{1} \mathrm{H}\right\}, 75 \mathrm{MHz}, \mathrm{CDCl}_{3}\right): \delta 172.50,139.66,133.09$, $129.92,128.81,127.90,60.36,34.05,25.02,14.32$.

\section{Results and Discussion}

\subsection{Synthesis}

Herein, we are presenting an efficient procedure for the synthesis of 1,2-bis(bis(4-chlorophenyl) methyl) diselane (1) by the reduction of elemental selenium with hydrazine hydrate followed by alkylation using 4,4'-dichloro diphenylmethyl chloride. Studies indicate that out of all the reducing agent known for reduction of selenium viz., alkali metals in ammonia, samarium iodide, rongalite and $\mathrm{NaOH} / \mathrm{PEG}-400$ etc., hydrazine hydrate was found to be most effective. [18] The diselenide ion $\mathrm{Se}_{2}^{2-}$ generated by the reduction of selenium using alkaline hydrazine hydrate in DMF upon in situ alkylation affords the 1,2-bis(bis(4-chlorophenyl)methyl) diselane in good yield (Scheme 1).

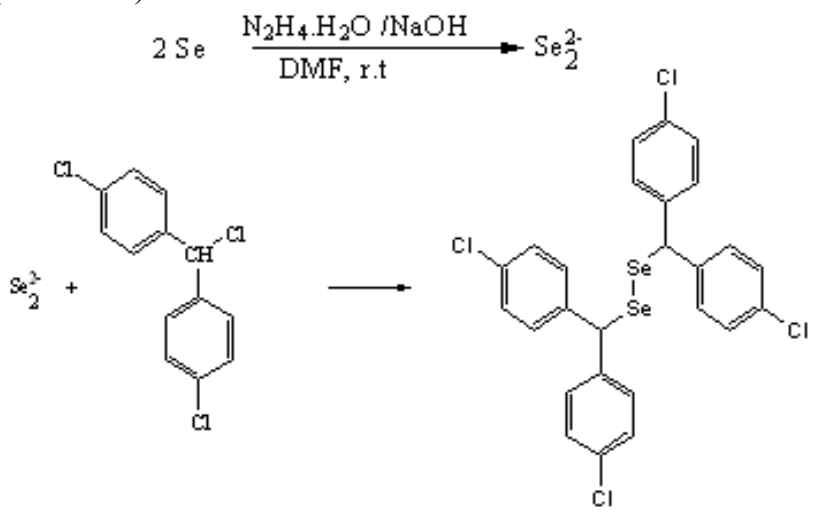

Scheme 1. Reaction Scheme for the synthesis of 1,2-bis[bis

(4-chlorophenyl)methyl] diselane

Under similar reaction conditions, an attempt has been made to prepare the corresponding ditelluride. However, it could not be isolated as its rapid detelluration leads to the formation of 1,1,2,2-tetraphenylethane. The instability of 1,2-bis(bis(4-chlorophenyl)methyl)ditellane can be attributed to low bond dissociation energy of a highly sterically constrained aliphatic C-Te bond.

We have recently reported the facile synthesis of phenyl/benzylselenoalkonates [19] and mono- and spiro $\beta$-lactam [20]. Taking a leaf out of this, we present here the synthesis of some novel selenoalkanoates (3i-3j). Starting substrate 1,2-bis(bis(4-chlorophenyl)methyl) diselane (1) was subjected to reductive cleavage with sodium borohydride for the synthesis of a variety of symmetrical/un symmetrical dichloro diphenylmethyl selenides (Scheme 2). 


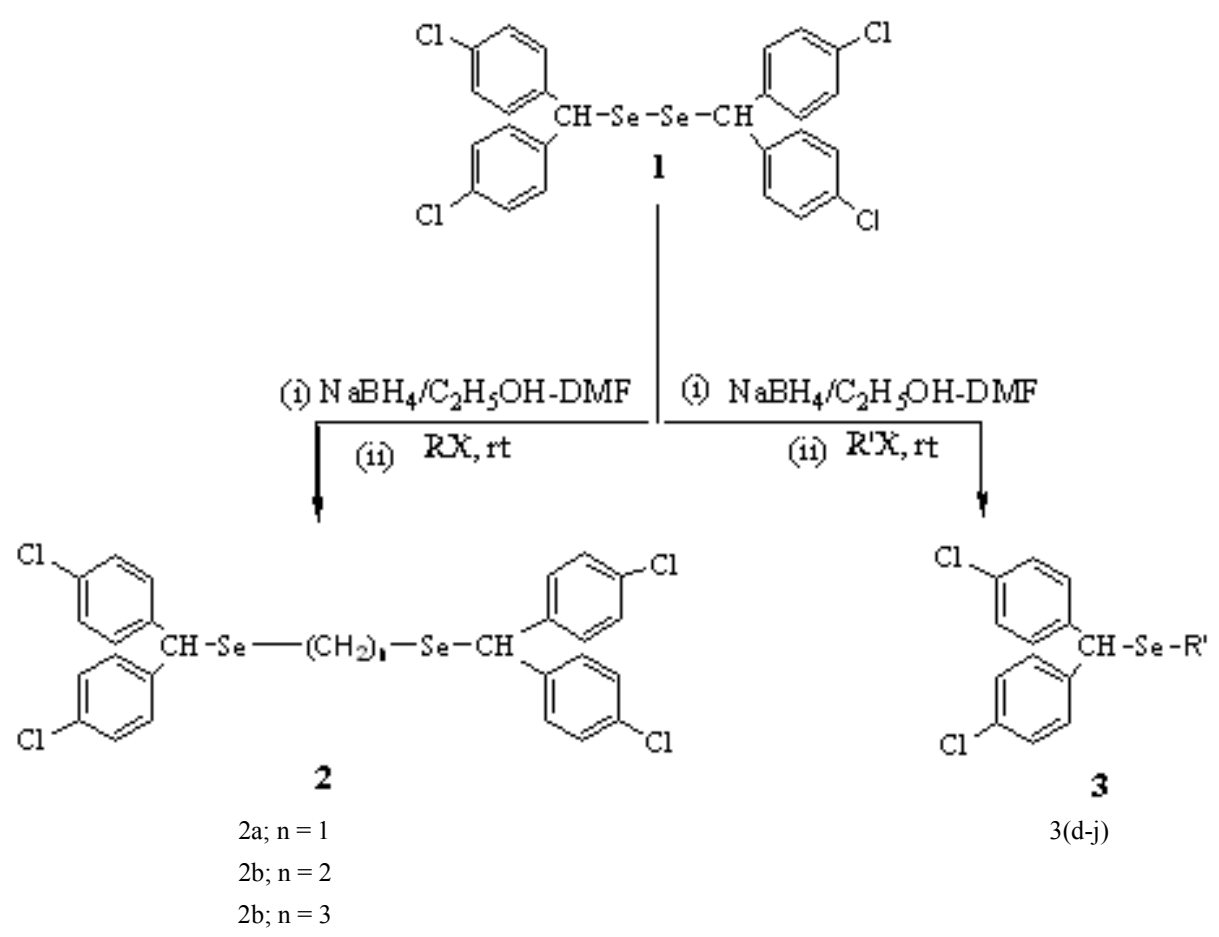

Scheme 2. Reaction scheme for the synthesis of derivatives of 1,2-bis[bis(4-chlorophenyl) methyl] diselane

Table 1. Various symmetrical and unsymmetrical compounds synthesized by using variety of alkylating reagent (RX).

\begin{tabular}{|ccccc|}
\hline $\begin{array}{c}\text { S. } \\
\text { No. }\end{array}$ & Substrate & $\mathrm{RX}$ & Product & $\begin{array}{c}\text { Yield } \\
(\%)\end{array}$ \\
1. & $\mathbf{1}$ & $\mathrm{CH}_{2} \mathrm{I}_{2}$ & $\mathbf{2 a}$ & 74 \\
2. & $\mathbf{1}$ & $\mathrm{Br}\left(\mathrm{CH}_{2}\right)_{2} \mathrm{Br}$ & $\mathbf{2 b}$ & 89 \\
3. & $\mathbf{1}$ & $\mathrm{Br}\left(\mathrm{CH}_{2}\right)_{3} \mathrm{Br}$ & $\mathbf{2 c}$ & 63 \\
4. & $\mathbf{1}$ & $\mathrm{CH}_{3} \mathrm{I}$ & $\mathbf{3 d}$ & 54 \\
5. & $\mathbf{1}$ & $\mathrm{CH}_{3} \mathrm{CH}_{2} \mathrm{Br}$ & $\mathbf{3 e}$ & 78 \\
6. & $\mathbf{1}$ & $\mathrm{BrCH}_{2} \mathrm{CH}_{2} \mathrm{CH}_{2}$ & $\mathbf{3 f}$ & 52 \\
7. & $\mathbf{1}$ & $\mathrm{CH}_{3} \mathrm{CH}_{2} \mathrm{CH}_{2} \mathrm{CH}_{2} \mathrm{Br}_{2}$ & $\mathbf{3 g}$ & 63 \\
8. & $\mathbf{1}$ & $\mathrm{ClCH}_{2} \mathrm{COOCH}_{2} \mathrm{CH}_{3}$ & $\mathbf{3 h}$ & 78 \\
9. & $\mathbf{1}$ & $\mathrm{ClCH}_{2} \mathrm{CH}_{2} \mathrm{COOCH}_{2} \mathrm{CH}_{3}$ & $\mathbf{3 i}$ & 65 \\
10. & $\mathbf{1}$ & $\mathrm{ClCH}_{2} \mathrm{CH}_{2} \mathrm{CH}_{2} \mathrm{COOCH}_{2} \mathrm{CH}_{3}$ & $\mathbf{3 j}$ & 50 \\
\hline
\end{tabular}

The diselenide was made completely soluble using ethanol as a solvent. The in situ generation of selenolate ion forms a copious precipitates due to the poor solubility of borane complexed selenolate ion $\left[\left(\mathrm{Ph}_{2} \mathrm{CHSe}\right) \mathrm{B}\left(\mathrm{OC}_{2} \mathrm{H}_{5}\right)_{3}\right]^{-}$[21] However, use of dimethyl formamide (DMF) as a co-solvent, improved the yield of the unsymmetrical 1,2-bis(bis(4-chlorophenyl)methyl) selenides to two folds in most cases, mainly by solubilizing the 1,2-bis(bis(4-chlorophenyl)methyl) diselane ion [22] besides increasing their nucleophilicity. Dimethyl formamide being a highly polar solvent has been removed by simply washing with water.

It is interesting to note that most of these n-alkyl dichloro diphenyl methyl selenides (3d-3j) prepared undergo decomposition over a period of time to form symmetrical dialkyl diselenides and 1,1,2,2,4- tetraphenyl ethane with traces the elemental selenium. This decomposition probably occurs by a radical mechanism that involves the cleavage of $\mathrm{C}$-Se and Se-Se bond (Scheme 3). This is in agreement with the thermal and photochemical decomposition behaviour of dibenzyl diselenide and bis(4-diphenylmethyl) diselenide [23]. The primary process is presumed to involve the homolytic cleavage of $\mathrm{C}$-Se bond towards dichloro diphenylmethyl carbon.

\subsection{Spectroscopic Studies}

All the compounds prepared were characterized by various spectroscopic techniques viz., ${ }^{1} \mathrm{H}$ NMR, ${ }^{13} \mathrm{C}\left\{{ }^{1} \mathrm{H}\right\}$ NMR, ${ }^{77} \mathrm{Se}\left\{{ }^{1} \mathrm{H}\right\}$ NMR and IR. spectroscopy. In the ${ }^{1} \mathrm{H}$ NMR spectra of these unsymmetrical dichloro diphenylmethyl selenides prepared, the methine protons resonate in the range of $\delta 5.12-5.51 \mathrm{ppm}$ whereas, the aromatic proton display three different sets, two of which are multiplets appearing at $\delta 7.30-7.27$ and $\delta 7.25-7.24 \mathrm{ppm}$ while the third appears as a triplet in the region $\delta$ 7.21-7.20 ppm. However in symmetrical compounds, the methine proton shifts upward and the aromatic protons remain unaffected.

\section{3. ${ }^{77}$ Se-NMR Spectroscopic Analysis}

Selenium atom is very sensitive to extremely small electronic and steric changes. Therefore, its chemical shift is a suitable tool to probe its chemical environment within the molecule. ${ }^{77} \mathrm{Se}\left\{{ }^{1} \mathrm{H}\right\}$ NMR was recorded in $\mathrm{CDCl}_{3}$ employing dimethyl selenide as an external reference resonating at $0(\delta$, $\mathrm{ppm})$. The selenium signal for compounds (1), (2c), (3d), (3f), and (3i) appears at $\delta 528.5,339.1,456.6,251.1$, and $305.5 \mathrm{ppm}$ respectively 


\subsection{Mass Spectroscopic Analysis (EI-MS)}

The mass spectra of all the compounds prepared are rich but complicated due to several isotopes of selenium and their different possible fragments. For most compounds analyzed, the molecular ion peak is very weak. The fragment corresponding to the mass of dichloro diphenylmethyl radical is most intense and appears invariably at 234 , and exhibits the mixed dichloro pattern as well. The fragment ions containing selenium show a highly characteristic and definite pattern of signal intensities depending on the natural abundance of its various isotopes of selenium.

\section{Conclusions}

In this paper authors have used a convenient methodology for the synthesis of hitherto unknown 1,2-bis[bis(4-chlorophenyl)methyl]diselane and further a reductive cleavage of $\mathrm{Se}-\mathrm{Se}$ bond of 1,2-bis[bis(4-chlorophenyl)methyl]diselane with sodium borohydride to generate selenide anion to synthesis variety of symmetrical and unsymmetrical analogues.

\section{Acknowledgements}

We gratefully acknowledge the financial support for this work from the Council of Scientific and Industrial Research (CSIR), New Delhi, India.

\section{REFERENCES}

[1] S. Patai, Z. Rappoport, Eds.; The Chemistry of Organic Selenium and Tellurium Compounds, John Wiley, Chichester. 1986, Vol.1.

[2] D.L. Klayman, W.H.H. Günther, Organic selenium Compounds: Their Chemistry and Biology, John Wiley \& Sons: New York. 1973.

[3] B. Krebs, F.P. Alhers, Developments in chalcogen-halide chemistry, Adv. Inorg. Chem. 1990, 35, 235-317.

[4] E.E. Aynsley, N.N. Greenwood, J.B. Leach, $\mathrm{Zn} / \mathrm{ZrCl}_{4}$ system induced reductive cleavage of $\mathrm{Se}$-Se bond in diaryl diselenide. A novel method for the synthesis of selenoesters, Chem. Ind. 1966, 376-381.

[5] Y.K. Lin, D.Q. Xu, Z.Y. Xu, Y.M. Zhang, A convenient and steroselective synthesis of (Z)-allyl selenides via $\mathrm{Sm} / \mathrm{TMSCl}$ system promoted coupling of Baylis-Hillman adducts with diselenides. J. Zhejiang Uni Sci B. 2006, 7, 393- 396.

[6] K.K. Bhasin, S. Doomra, G. Kaur, E. Arora, N. Singh, Y. Nagpal, R. Kumar, Rishu, T.M. Klapoetke, S.K. Mehta, Synthesis of unsymmetrical pyridyl aryl selenides by the reductive cleavage of Se-Se bond, Phosphorus, Sulfur, and Silicon. 2008, 183, 992-997.

[7] H. Suzuki, M. Yoshinaga, K. Takaoka, Y. Hiroi, A simple synthesis of aryl difluromethyl selenides and tellurides, Synthesis. 1985, 497-499.

[8] H.J. Reich, J.M. Renga, L.L. Reich, Organoselenium chemistry. Conversion of ketones and enones by selenoxide syn elimination, J. Am. Chem. Soc. 1975, 97, 5434-5447.

[9] K.K. Bhasin, Newer aspects in synthetic methodolgy of pyridyl chalcogen $(\mathrm{E}=\mathrm{Se} / \mathrm{Te})$ compounds. Phosphorus, Sulfur, and Silicon and the related elements, 2005, 180, 1063-1070.

[10] R.K. Das, S. U. Hossain, S. Bhattacharya, Protective effect of diphenylmethyl selenocynate against $\mathrm{CCl}_{4}$-induced hepatic injury, 2007, 27, 527-537.

[11] D.S. Margolis, R.W. Pittman, The product of interaction of hydrogen selenide ketones, J. Chem. Soc. 1957 799-805.

[12] K. Okuma, J. Sakata, Y. Tacchibana, T. Honda, H. Ohta, Stereoselective synthesis of trans olefins by the reaction of Wittig reagents with selenium.Formation of selenocarbonyl compounds, Tetrahedron Lett. 1987, 28, 6649-6652.

[13] T. Shimizu, D. Miyasaka, N. Kamigata, Reaction of 1,3Bis(alkylseleno)allenes with diphenyl diazomethane, J. Org. Chem. 2001, 66, 7202-7204.

[14] K.K. Bhasin, N. Singh, R. Kumar, D. Gupta, S.K. Mehta, T.M. Klapoetke, M.-J. Crawford, A convenient synthesis of some symmetricala and unsymmetrical diarylmethyl sulfur and selenium compounds: X-ray crystal struture of diphenylseleno-2-propene and bis[p-chloro phenyl (phenylmethyl]diselenide., J. Organomet. Chem. 2004, 689, 3327-3334.

[15] K.K. Bhasin, B.S. Bhandal, J. Singh, N. Singh, K.N. Singh, P. Singh, A new and convenient route to 2,2'-dipyridyl diselenide/ditelluride ans some of their alkyl/aryl derivatives through $\mathrm{BF}_{3}$-complexed pyridyl carbanion, Syth. Commu. 2002, 32, 1319- 1325

[16] K.K. Bhasin, A. Sandhu, R.D. Verma, Synthesis and characterization of new alkoxyalklorganyl selenides,Synth. React. Met.-Org. Chem. 1988, 18, 141-151.

[17] W.S Haller, K.J. Irgolic, Diaryl tellurides from Grignard reagents and elemental tellurium, J. Organomet. Chem. 1972, $38,97-103$.

[18] L.F. Audrieth, B.A. Ogg, The Chemistry of Hydrazine, John Wiley\& Sons Inc., New York, 1951.

[19] A. Bhalla, S. Sharma, K.K. Bhasin, S.S. Bari, Convenient preparation and benzylseleno- and phenylselenoalkonic acids: Reagents for synthesis of organoselenium compounds, Synthetic Commu. 2007, 37, 783-793.

[20] A. Bhalla, P. Venugopalan, K.K. Bhasin, S.S. Bari, Seleno- $\beta$-lactams: Synthesis of monocyclic and spirocyclic selenoazetidin-2-ones, Tetrahedron, 2007, 63, 3195-3204.

[21] D.L. Klaymann, T.S. Griffin, Reaction of selenium with sodium borohydride in protic solvents. A facile method for the introduction of selenium into organic molecules, $\mathrm{J}$. Am. Chem. Soc. 1973, 95, 197-199.

[22] D. Liotta, U. Sunnay, H. Santiestiban, W. Marckienicz, The generation of uncomplexed phenyl selenide anion and its applicability to $\mathrm{S}_{2} \mathrm{~N}$-type ester clevages, Tetrahedron Lett. 1977, 50, 4365-4367.

[23] J.Y.C. Chu, D.G. Marsh, J.W. Lewicki, I.L. Weaver, Photochemistry of organo chalcogen compound. 3. 
Photochemistry of Bis(benzoylmethyl) tellurium dichlorides, J. Am. Chem. Soc. 1976, 98, 8432-8437.
[24] A. I. Vogel, Textbook of Quantitive Inorganic Analysis, IV Ed. ELBS, Longman, London. 1978, 477. 